\title{
Controlled and Reproducible Domain Wall Displacement by Current Pulses Injected into Ferromagnetic Ring Structures
}

\author{
M. Kläui, ${ }^{1}$ C. A. F. Vaz, ${ }^{2}$ J. A. C. Bland, ${ }^{2, *}$ W. Wernsdorfer,${ }^{3}$ G. Faini,${ }^{4}$ E. Cambril,${ }^{4}$ \\ L. J. Heyderman, ${ }^{5}$ F. Nolting, ${ }^{5}$ and U. Rüdiger ${ }^{1}$ \\ ${ }^{1}$ Fachbereich Physik, Universität Konstanz, Universitätsstrasse 10, D-78457 Konstanz, Germany \\ ${ }^{2}$ Cavendish Laboratory, University of Cambridge, Madingley Road, Cambridge, CB3 OHE, United Kingdom \\ ${ }^{3}$ Laboratoire L. Néel-CNRS, BP 138, 38274 Grenoble, France \\ ${ }^{4}$ Laboratoire de Photonique et de Nanostructures-CNRS, Route de Nozay, 91460 Marcoussis, France \\ ${ }^{5}$ Paul Scherrer Institut, CH-5232 Villigen PSI, Switzerland
}

(Received 22 April 2004; published 15 March 2005)

\begin{abstract}
In a combined numerical and experimental study, we demonstrate that current pulses of different polarity can reversibly and controllably displace a magnetic domain wall (DW) in submicrometer permalloy $(\mathrm{NiFe})$ ring structures. The critical current densities for DW displacement are correlated with the specific spin structure of the DWs and are compared to results of micromagnetic simulations including a spin-torque term. Using a notch, an attractive local pinning potential is created for the DW resulting in a highly reproducible spin structure of the DW, critical for reliable current-induced switching.
\end{abstract}

\section{DOI: 10.1103/PhysRevLett.94.106601}

Switching by domain wall motion [1] induced by spinpolarized currents rather than by external fields is a promising approach to the switching of magnetic nanostructures, since it entails simple fabrication processes without the need for strip lines, combined with the possibility of achieving fast and reproducible switching [2-6]. The current-induced magnetization switching mechanism has been shown to be able to switch multilayer giant magnetoresistance structures [7] and to reverse simple single layer elements as observed for $L$-shaped elements [2,3], for ring-shaped [4] and straight [5] structures with constrictions, and also in multilayer wires [6]. This currentinduced domain wall motion is due to a spin-torque effect, where the electrons transfer angular momentum to the domain wall when passing through it, pushing it in the direction of the electron flow [8]. Since the original paper by Berger [8], a number of different theories have been suggested that treat the interaction between the spinpolarized current and the magnetization in the ballistic limit $[9,10]$ or in the diffusive limit $[9,11]$. For wide inplane domain walls with widths of hundreds of nanometers [12] the spins of the electrons are expected to follow the magnetization adiabatically, and thus the diffusive description is expected to apply. While there are a number of experimental results, no direct quantitative comparison to the theoretical predictions has been made available.

In this Letter we demonstrate that different types of head-to-head domain walls present in ring structures can be reversibly and controllably displaced by spin-polarized current pulses. Direct comparison with the results of our micromagnetic simulations that include a diffusive spintorque term allows us to determine to what extent the experimentally observed effects can be described by a purely diffusive spin-torque theory.

Rings are a particularly apt geometry to investigate the effect of pulses on domain walls, since head-to-head walls
PACS numbers: 72.15.Gd, 75.60.Ch, 75.60.Ej, 85.70.Kh

with different spin structures can be obtained for different ring geometries [12]. For thin film rings, transverse walls [Fig. 1(c)] are observed, while in thick rings vortex walls [Fig. 1(d)] are present [13]. Because of the constant curvature, the walls can easily be positioned by applying a field

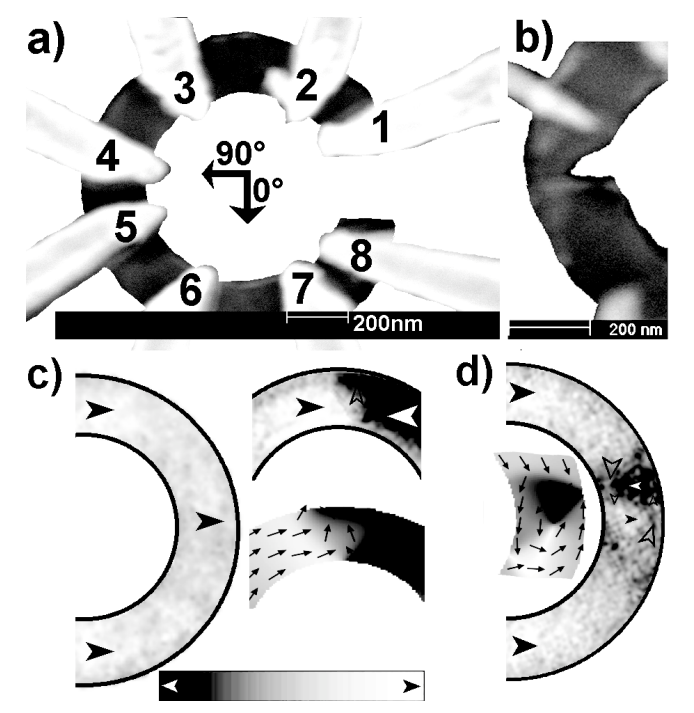

FIG. 1. SEM image of a ring structure, outer diameter $950 \mathrm{~nm}$, inner diameter $550 \mathrm{~nm}$, thickness $17 \mathrm{~nm}$ permalloy (the arrows indicate the direction convention used). (b) Detail of a ring of the same geometry (10 nm film thickness) with a notch. (c) PEEM images of transverse head-to-head domain walls at two different positions in a ring with the same geometry as in (a) but with $10 \mathrm{~nm}$ film thickness and without contacts and gap. A corresponding simulation calculated using the OOMMF package $[12,27]\left(M_{S}=800 \times 10^{3} \mathrm{~A} / \mathrm{m}, A=13 \times 10^{-12} \mathrm{~J} / \mathrm{m}, \quad K_{1}=\right.$ $0 \mathrm{~J} / \mathrm{m}^{3}$, cell size: $4 \mathrm{~nm}$ ) is shown for comparison. The magnetization directions are given by the grey-scale bar. (d) PEEM image and micromagnetic simulation of a vortex head-to-head domain wall in a $34 \mathrm{~nm}$ thick ring. 
along the desired direction $[14,15]$. The permalloy (NiFe) ring structures 5 to $34 \mathrm{~nm}$ thick with $2 \mathrm{~nm}$ Au capping and submicrometer lateral dimensions used for this experiment were fabricated as described in Ref. [16]. A scanning electron microscopy (SEM) image is presented in Fig. 1(a). For applications the domain wall position must be read out by electrical means, and thus we use standard four-probe magnetoresistance measurements (to get a maximum of flexibility, eight nonmagnetic contacts are symmetrically distributed around the ring, numbered 1 to 8) [16]. The ring structure is severed between contacts 1 and 8 to make sure that the resistance measured between any two contacts corresponds only to the resistance of the area between the contacts. As shown earlier $[15,16]$, the presence of a domain wall between two contacts can easily be sensed by measuring the resistance. Since the magnetoresistance is dominated by the anisotropic magnetoresistance, a maximum resistance is found if the spins are parallel (or antiparallel) to the injected current. This corresponds to a state when no domain wall is present between two contacts and the magnetization follows the direction of the perimeter of the ring [15]. If a domain wall is present between the contacts, some of the magnetization in the domain wall points perpendicularly to the current, and hence the resistance is lowered $[15,16]$. In this experiment the voltage was measured using a lock-in amplifier first between contacts 6 and 7 (see Fig. 1), the ac lock-in current was injected at contact 1 , the current pulses were injected at contact 2 , and contact 8 was grounded. We use current pulses of up to $20 \mu$ s width and current densities up to $5 \times 10^{12} \mathrm{~A} / \mathrm{m}^{2}$ (such high current densities would destroy the ring if used as continuous currents, which also have the drawback of electromigration and strong heating, reducing switching fields and thus stability $[2,4]$ ). The pulses could be triggered by hand, and the effect on the magnetoresistance was monitored concurrently. First, we determine the resistance as a function of domain wall position without injecting current pulses. Figure 2(a) shows the resistance at remanence after the ring has been saturated with a field applied along the direction given by the angle indicated on the abscissa (see also Fig. 1). After relaxing the field, the domain wall is then located at a position in the direction the field was applied, as shown in Ref. [15]. For directions between $0^{\circ}$ and $5^{\circ}$, the resistance is low (level $A$ ), corresponding to the domain wall being located between contacts 6 and 7 . Above $6^{\circ}$ the resistance is high (level $B$ ), corresponding to the domain wall being located outside the area between the contacts as schematically shown in Fig. 2(c). To investigate the influence of a current pulse on a domain wall, we have first placed a domain wall between contacts 6 and 7 by saturating the ring with a field along $0^{\circ}$ (see Fig. 1) and relaxation of the field to zero. Resistance level $A$ corresponds thus to the head-to-head domain wall being located between these contacts. At $t=$ $t_{1}$ we then inject a positive current pulse (current density of $2 \times 10^{12} \mathrm{~A} / \mathrm{m}^{2}$; positive currents mean that the electrons flow from contact 8 to contact 2). After the current pulse,

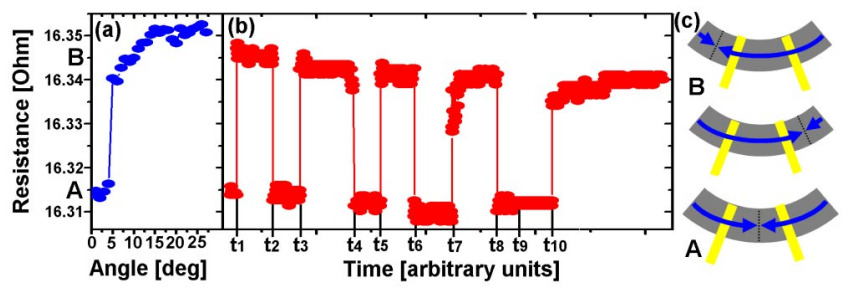

FIG. 2 (color online). (a) Remanent resistance vs domain wall position for the (17 nm thick) ring of Fig. 1(a). Level $A$ corresponds to the domain wall being located between contacts 6 and 7 , level $B$ to the domain wall being located outside. (b) Resistance as current pulses are injected with the domain wall initially located between the contacts (before $t_{1}$ ). (c) Schematics for the domain wall to the left of the contacts (top), to the right (middle), and between the contacts (bottom). The top configuration corresponds to the resistance level $B$ attained after the current pulses at $t_{1}, t_{3}, t_{5}, t_{7}$, the middle configuration is obtained after $t_{10}$, and the bottom configuration before $t_{1}$ and after $t_{2}, t_{4}, t_{6}, t_{8}$ corresponding to resistance level $A$.

the resistance has increased to level $B$, meaning that the domain wall has left the area between the contacts and is now located outside, to the left of contact 6 . When at $t=t_{2}$ we apply a negative current pulse with the same amplitude, the domain wall is pushed back into the area flanked by contacts 6 and 7 and the resistance jumps back down to level $A$ [Fig. 2(b)]. This same current pulse sequence is now repeated a few times $\left(t_{3}\right.$ to $\left.t_{8}\right)$ and the resistance levels $A$ and $B$ are reversibly and reproducibly attained corresponding to the domain wall being moved in and out of the area between contacts 6 and 7. At $t_{8}, t_{9}$, and $t_{10}$ we then apply three consecutive negative current pulses. The first current pulse moves the domain wall back into the area between the contacts (corresponding to the resistance dropping to level $A$ ). The second current pulse has no effect on the resistance, meaning that the domain wall is still inside the area between the contacts. After the third negative pulse at $t_{10}$ the resistance has increased, meaning that the domain wall has been pushed out of the area between the contacts (this time to the right of contact 7).

For device applications, the switching between two stable states has to be extremely robust. In the contact configuration mentioned above, the domain wall can propagate between contacts 2 and 8 , since the current pulses are injected between these contacts. This can pose problems, since the domain wall can overshoot the desired traveling distance as was actually observed in some of the samples for different pulse heights and widths. In order to prevent unintentional overshooting, an overlapping configuration of the contacts can be used: in the following experiment we measure the resistance between contacts 2 and 4 and inject the current pulses at contact 3 , while contact 1 is grounded (the lock-in current is injected at contact 8). Now the domain wall can be moved by a pulse only between contacts 3 and 1 regardless of the pulse height and length, which leads to very robust switching. Using this overlapping contact configuration, a set of switching cycles 
with the domain wall being moved by negative and positive current pulses between positions $\alpha$ (low resistance; domain wall between contacts 2 and 3 ) and $\beta$ (high resistance; domain wall between contacts 1 and 2) is shown in Fig. 3 for a ring with identical geometry as that in Fig. 1 but with $10 \mathrm{~nm}$ film thickness. At $t=t_{1}$ we inject four consecutive positive pulses (electrons flowing from contact 1 to contact 3) with no effect on the resistance, since the domain wall cannot move beyond contact 3. A negative pulse at $t=t_{2}$ moves the domain wall back to position $\beta$ outside the area between the contacts, and the resistance increases consequently. Furthermore, as expected, a domain wall positioned between contacts 3 and 4 is not affected by the current pulses, which means that the domain wall propagation distance can be controlled by choosing an appropriate contact configuration. For applications this effect overcomes the problem of uniformity and reproducibility of the critical switching current of a large array of elements, since every element can be individually switched with the same current pulse (which simply needs to be higher than that for the element with the highest switching current). In order to understand the interaction between the domain wall and the current pulses, we have imaged the domain walls using photoemission electron microscopy (PEEM) [12,17] as presented in Figs. 1(c) and 1(d). The 5-10 nm thick rings exhibit primarily transverse head-tohead domain walls [Fig. 1(c)], whereas for 17-34 nm thick rings, the domain wall is a vortex wall [Fig. 1(d)], and as seen in Figs. 2 and 3 both wall types can be displaced by a current pulse (for the ring geometry investigated here, the transition between the two domain wall types lies between 10 and $17 \mathrm{~nm}$ [18]). In Fig. 4 the critical current needed to displace the domain wall in rings with the same geometry presented in Fig. 1 is shown as a function of film thickness. It can be seen that the critical current density is not constant but rather varies with film thickness, increasing abruptly at the thickness range between 10 and $17 \mathrm{~nm}$,

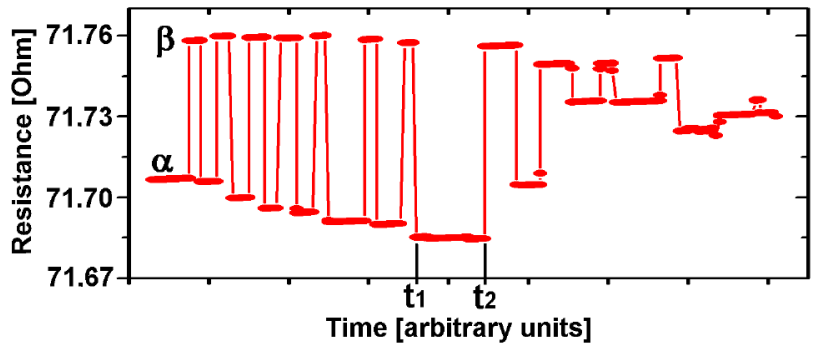

FIG. 3 (color online). The resistance is shown for a ring with the same geometry as in Fig. 1(a) but with a film thickness of $10 \mathrm{~nm}$ and an overlapping contact configuration. The domain wall is initially placed between contacts 2 and 3 by a magnetic field. The resistance is then switched between levels $\alpha$ and $\beta$ by injecting current pulses of opposite polarity corresponding to the domain wall being moved between the area flanked by contacts 2 and $3(\alpha)$ and the area flanked by contacts 1 and $2(\beta)$. After $t_{2}$ the domain wall stays halfway underneath contact 2 corresponding to a medium resistance. exactly at the transition thickness between the transverse and the vortex wall regimes.

Since in the case of such wide domain walls the spins of the conduction electrons are expected to follow the local magnetization adiabatically, we have modeled the influence of the current on the domain wall, as a first approach, by adding a diffusive term $u(\partial m / \partial x)$ to the LandauLifshitz-Gilbert equation that describes the time evolution of the magnetization: $\dot{m}=\gamma H \times m+\alpha m \times \dot{m}-$ $u(\partial m) / \partial x$ with $\gamma$ the gyromagnetic ratio, $\alpha$ the damping constant, and $x$ the local direction of the current flow $[11,19]$. The "equivalent velocity" $u$ is given by $u=$ $\frac{J P g \mu_{B}}{2 e M_{s}}$ with $J$ the current density and $P$ the current polarization (assumed to be 0.4 for permalloy [20], the other parameters used for the simulations are given in the caption of Fig. 1). Micromagnetic simulations including this diffusive term (using the micromagnetics simulator $[18,21]$ and by other groups [19]) show that for currents below a critical current density, even in a perfect wire with no edge roughness, domain walls do not move but are just deformed $[18,19]$. In this adiabatic model domain wall movement sets in only above a critical current density $[19,22]$. Comparing the experimental critical current densities (red dots in Fig. 4) with the calculated values (black squares), we see that qualitatively the jump in the critical current density when the domain wall type changes from transverse to vortex is reproduced by the simulations. Quantitatively the critical current density is reproduced well for transverse walls, but for vortex walls the values obtained using this purely diffusive adiabatic description are higher than the experimental values. This fact is quite surprising, since it might indicate that, even for these very wide domain walls, the electron transport is not completely diffusive, but a mistracking between the local magnetiza-

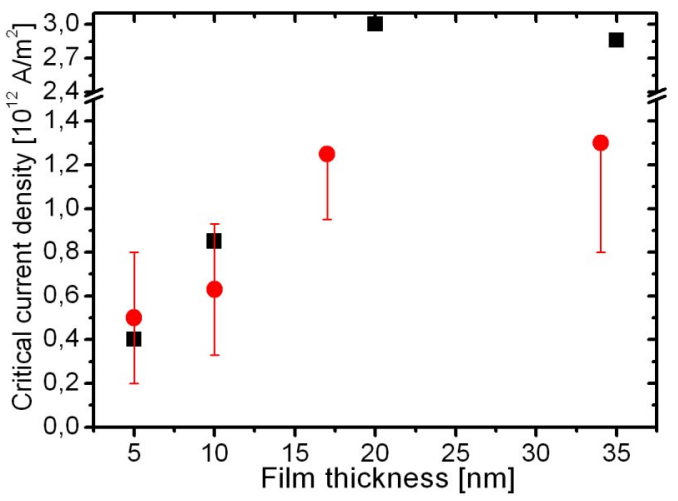

FIG. 4 (color online). The critical current density for domain wall motion is shown for the rings of Fig. 1(a) with different film thicknesses (red circles). The jump between 10 and $17 \mathrm{~nm}$ is associated with the change in domain wall spin structure from transverse to vortex. Calculated critical current densities are shown as black squares. In addition to the jump, an increase in the critical current density for increasing film thickness could be expected, since the coercivity and the field needed to move a domain wall increase with increasing film thickness. 
tion direction and the electron spin direction might exist. In order to reproduce the experimental results quantitatively the theory needs to be revised to treat nonadiabatic effects; edge irregularities might also play a role [22]. The reason that the discrepancy becomes evident for vortex walls could lie in the large angles between adjacent spins around the vortex core, where the diffusive adiabatic description might not be sufficient.

Finally, the part of the graph after $t=t_{2}$ in Fig. 3 shows an important trend, observed in all samples after a few to tens of switching cycles: the alternating current pulses from $t=t_{2}$ onwards have less and less of an effect on the resistance, which levels off at around half the resistance between the two domain wall positions $\alpha$ and $\beta$, meaning that the domain wall is positioned just underneath contact 2 and is thereafter not significantly affected by the alternating current pulses.

The reason for this behavior must lie in a change in the internal spin structure of the domain wall by the current pulse, so that the wall becomes less susceptible to subsequent pulses (e.g., a widening of the domain wall, since the effect should depend on the wall spin structure $[18,23]$ ). This is corroborated by the fact that domain walls that are located between the contacts after a large number of pulses can show a reduced resistance, which is the signature of an increased transverse magnetization component inside the wall possibly due to a widening of the wall $[15,24]$. An example for this behavior can be seen in Fig. 3. The original resistance is retrieved when the original spin structure of the domain wall is restored by again applying an external field. Reasons for the deformation of the wall may be both the Oersted field of the large current pulses and the spin-torque effect induced deformation $[22,25]$. To circumvent the problem of domain wall deformation, a ring with a notch was designed as seen in Fig. 1(b). Notches have been found to create attractive potential wells for domain walls $[15,26]$, which adopt a spin structure at the constriction that lowers its energy. Experimentally this is confirmed in a ring with the notch shown in Fig. 1(b). The domain wall is positioned at different locations outside the notch and, then it is subjected to a variety of current pulses of varying intensity. The resistance is measured when the wall is finally positioned at the notch. The resistance is found to coincide every time within $0.005 \%$ (as compared to a change of $0.03 \%$ in Fig. 3) to the resistance obtained by placing the wall into the notch with a field, thereby demonstrating that the spin structure of the domain wall is reproducibly restored when it enters the notch, even if the domain wall had been deformed beforehand. This special "repair mechanism" for the spin structure of the domain wall could pave a way for reproducible currentinduced magnetization switching that could lead to reliable memory elements with unlimited switching cycles.

In conclusion, using a special contact geometry we have demonstrated that we can controllably displace vortex and transverse head-to-head domain walls in submicrometer permalloy ring structures by using current pulses and without any externally applied magnetic fields. The domain walls move in the direction of the electron flow, consistent with the spin-torque effect [8]. The critical current densities for displacement are lower for transverse walls, in agreement with the results of micromagnetic simulations, while the calculations predict a larger critical current density for vortex walls suggesting that, surprisingly for wall widths of hundreds of nanometers, our purely diffusive description of the spin-torque effect is not sufficient. Finally, reliable switching can be achieved by including notches to obtain a reproducible spin structure of the domain walls.

This work was supported by the CMI Magnetoelectronic Devices project, the "Region Ile de France," the "Conseil General de l'Essonne," and the "Deutsche Forschungsgemeinschaft" (SFB 513). Part of this work was carried out at the Swiss Light Source, Paul Scherrer Institut (Villigen, Switzerland).

*Corresponding author.

Electronic address: jacb1@phy.cam.ac.uk

[1] D. A. Allwood et al., Science 296, 2003 (2002).

[2] N. Vernier, D. A. Allwood, D. Atkinson, M. D. Cooke, and R. P. Cowburn, Europhys. Lett. 65, 526 (2004).

[3] A. Yamaguchi et al., Phys. Rev. Lett. 92, 077205 (2004).

[4] M. Kläui et al., Appl. Phys. Lett. 83, 105 (2003).

[5] M. Tsoi, R. E. Fontana, and S. S. P. Parkin, Appl. Phys. Lett. 83, 2617 (2003).

[6] J. Grollier et al., Appl. Phys. Lett. 83, 509 (2003).

[7] J. A. Katine et al., Phys. Rev. Lett. 84, 3149 (2000); J.-E. Wegrowe et al., Europhys. Lett. 56, 748 (2001); J. Grollier et al., Appl. Phys. Lett. 78, 3663 (2001).

[8] L. Berger, J. Appl. Phys. 55, 1954 (1984).

[9] G. Tatara and H. Kohno, Phys. Rev. Lett. 92, 086601 (2004).

[10] V. A. Gopar et al., Phys. Rev. B 69, 014426 (2004).

[11] J. C. Slonczewski, J. Magn. Magn. Mater. 159, 11 (1996).

[12] M. Kläui et al., Phys. Rev. B 68, 134426 (2003); J. Phys. Condens. Matter 15, 985 (2003).

[13] M. Kläui et al., Appl. Phys. Lett. 85, 5637 (2004).

[14] J. Rothman et al., Phys. Rev. Lett. 86, 1098 (2001).

[15] M. Kläui et al., Phys. Rev. Lett. 90, 097202 (2003).

[16] M. Kläui et al., Appl. Phys. Lett. 81, 108 (2002).

[17] A. Locatelli et al., Surf. Rev. Lett. 9, 171 (2002).

[18] M. Kläui et al. (to be published).

[19] A. Thiaville et al., J. Appl. Phys. 95, 7049 (2004).

[20] B. Nadgorny et al., Phys. Rev. B 61, 3788 (2000).

[21] http://llgmicro.home.mindspring.com

[22] A. Thiaville et al., cond-mat/0407628; S. Zhang and Z. Li, Phys. Rev. Lett. 93, 127204 (2004).

[23] V. K. Dugaev, J. Berakdar, and J. Barnas, Phys. Rev. B 68, 104434 (2003).

[24] Recently the first direct evidence of domain wall spin structure modifications by spin currents has been obtained by spin-SEM measurements [18].

[25] X. Waintal and M. Viret, Europhys. Lett. 65, 427 (2004).

[26] M. Kläui et al., Physica (Amsterdam) 343B, 343 (2004).

[27] http://math.nist.gov/oommf. 